\title{
Fatal Rupture of a Giant Hydatid Cyst in an Elderly Patient
}

\author{
Ismail Altintop*, Mehmet Yilmaz, Zehra Alincak, Abdussamed Vural \\ Department of Emergency Medicine, Kayseri Training and Research Hospital, Kayseri, Turkey \\ Email: "draltintop1@hotmail.com
}

Received 30 April 2015; accepted 18 March 2016; published 21 March 2016

Copyright (C) 2016 by authors and Scientific Research Publishing Inc.

This work is licensed under the Creative Commons Attribution International License (CC BY). http://creativecommons.org/licenses/by/4.0/

c) (i) Open Access

\begin{abstract}
Hydatid cyst (HC) disease, which is endemic in Turkey, is mainly located in the liver. Cyst rupture is the most common complication of HC. Ultrasonography (US) and computerized tomography (CT) are the main diagnostic modalities for HC disease. Presented herein was a case of an elderly female patient who died shortly after presenting to the emergency department with severe abdominal pain. A giant HC was detected on initial evaluation by US. However, the presence of significant amounts of free fluid in the peritoneal space following a CT of the abdomen. The patient died despite medical and surgical intervention.
\end{abstract}

\section{Keywords}

Primary Hydatid Cyst, Hydatid Cyst Rupture, Acuteabdomen

\section{Introduction}

Hydatid cyst (HC) is caused by the tapeworm Echinococcus granulosus. Echinococcus granulosus is a cestode parasite (tapeworm) that causes hydatid infection.These cestodes have a worldwide distribution but the prevalence is higher in developing countries. This disease is endemic in cattle and sheep rearing regions of the world [1]. The parasitic infection is endemic in Turkey making it an important public health issue [2]. According to the data released by the Ministry of Health, 59,808 cases (3,518/year) and 939 deaths (55/year) were reported between 1987 and 2004 in Turkey. The liver and lungs are the most common locations for HCs, and affected patients usually remain asymptomatic for long periods before developing complications, the most frequent complication being cyst rupture [3]. HCs are mostly identified by radiological modalities.

\section{Case Presentation}

A 79-year-old woman was brought to the emergency department with a three-day history of worsening abdo-

\footnotetext{
${ }^{*}$ Corresponding author.
} 
minal pain and progressive clinical deterioration. The patient was responsive with a Glasgow coma score of 15 , but disoriented and agitated. She also had tachypnea and tachycardia, with a presenting blood pressure of 80/50 $\mathrm{mmHg}$ and oxygen saturation of $88 \%$ on room air. Intravenous hydration was initiated via a peripheral line. Physical examination of the chest was unremarkable with clear lung fields on auscultation. The patient's abdomen was tender to light palpation, especially in the right upper quadrant. Guarding and rebound tenderness were also present. Arterial blood gas analysis revealed the presence of metabolic acidosis. White blood count on hemogram was $11,520 / \mathrm{mm}^{3}$ with a platelet count of $337,000 / \mathrm{mm}^{3}$, hemoglobin concentration of $11.6 \mathrm{gr} / \mathrm{dL}$ and a hematocrit of 38.9\%. Results of serum biochemistry were as follows: glucose, $81 \mathrm{gr} / \mathrm{dL}$; BUN, $50 \mathrm{mg} / \mathrm{dL}$; creatinine, $2.7 \mathrm{mg} / \mathrm{dL}$; AST, $199 \mathrm{U} / \mathrm{L}$; ALT, $77 \mathrm{U} / \mathrm{L}$; Calcium, $9.3 \mathrm{mg} / \mathrm{dL}$; Sodium, $141 \mathrm{mmol} / \mathrm{dL}$; Potassium, 5.5 $\mathrm{mmol} / \mathrm{dL}$; Chloride, 98 mmol/dL; Amylase, $64 \mathrm{U} / \mathrm{L}$; CK, $161 \mathrm{mg} / \mathrm{dL}$; CK-MB, $24 \mathrm{U} / \mathrm{L}$.

An ultrasound performed for the acute abdomen revealed the presence of a large complicated cyst, $16 \times 15.5$ $\times 20 \mathrm{~cm}$ in size in the right lobe of the liver. The cyst had multiple septations and internal echoes with several smaller cystic formations within. Its posterior wall had calcifications with acoustic shadows. Another $6.5 \times 5 \mathrm{~cm}$ sized cystic mass with similar ultrasonographic features was observed posterior to the larger cyst. While the gallbladder and bile ducts appeared normal, a lymphadenopathy $17 \times 12 \mathrm{~mm}$ in size was observed in the portalhilum. No free peritoneal fluid could be ascertained.

Following surgical consultation, the patient was referred for a preoperative CT scan of the abdomen which confirmed the presence of an overall enlarged liver with a cystic mass $14 \times 13 \times 20 \mathrm{~cm}$ in size occupying a large portion of the hepatic right lobe (Figure 1). Another $9.5 \times 6 \mathrm{~cm}$ sized cyst was also detected in segment $6-7$ of the right lobe, which contained daughter vesicles consistent with type III hydatid cyst (Figure 2). A loculated fluid collection was observed adjacent to the larger cyst suggestive of leakage from the cyst. Free sub-diaphragmaticair was seen on the right, along with free intra-abdominal fluid in the peri-hepatic, sub-hepatic and lower abdominal spaces.

The patient was hypotensive during follow-up in the emergency department, with persistence of hemodynamic instability despite intravenous fluid resuscitation. She was promptly transferred to the anesthesiology intensive care unit. Emergency surgery was performed on the same day during which a cystotomy was followed by cystectomy and closure of the remnant cavity. Post-operatively, the patient continued to require mechanical ventilation and was followed-up in the ICU. She died 12 days after admission. Patient died from septic shock. We tried to obtain the images during surgery, but it has not reached the macroscopic picture.

\section{Discussion}

Echinococcus granulosus is a protozoa that attaches itself to the intestinal mucosa of carnivorous animals such

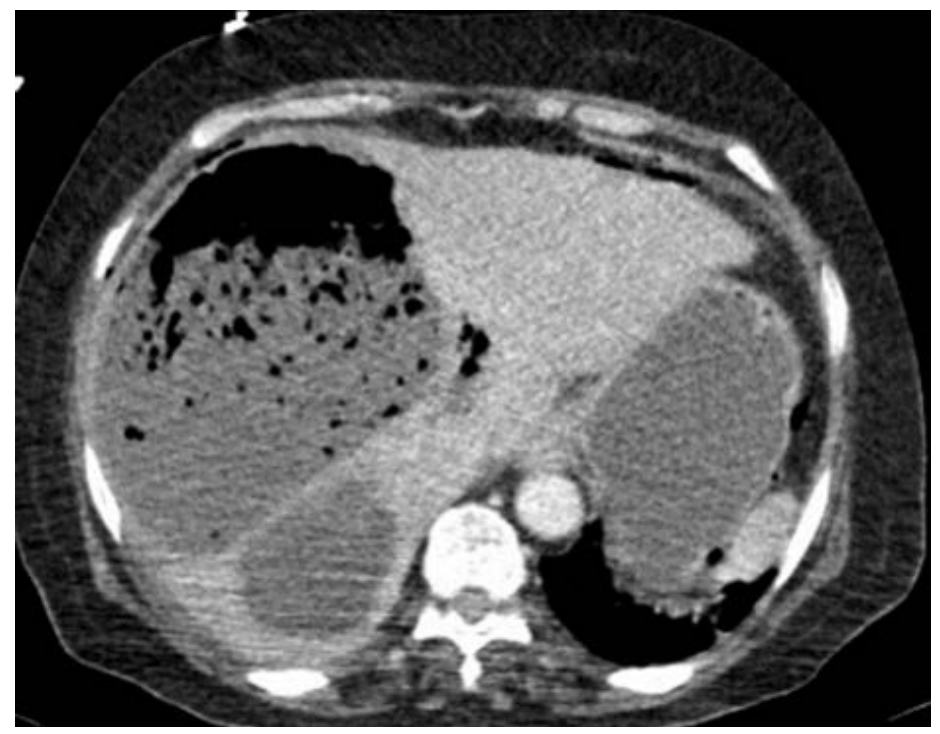

Figure 1. Computed tomography image; a cystic mass $14 \times 13 \times 20 \mathrm{~cm}$ in size occupying a large portion of the hepatic right lobe. 


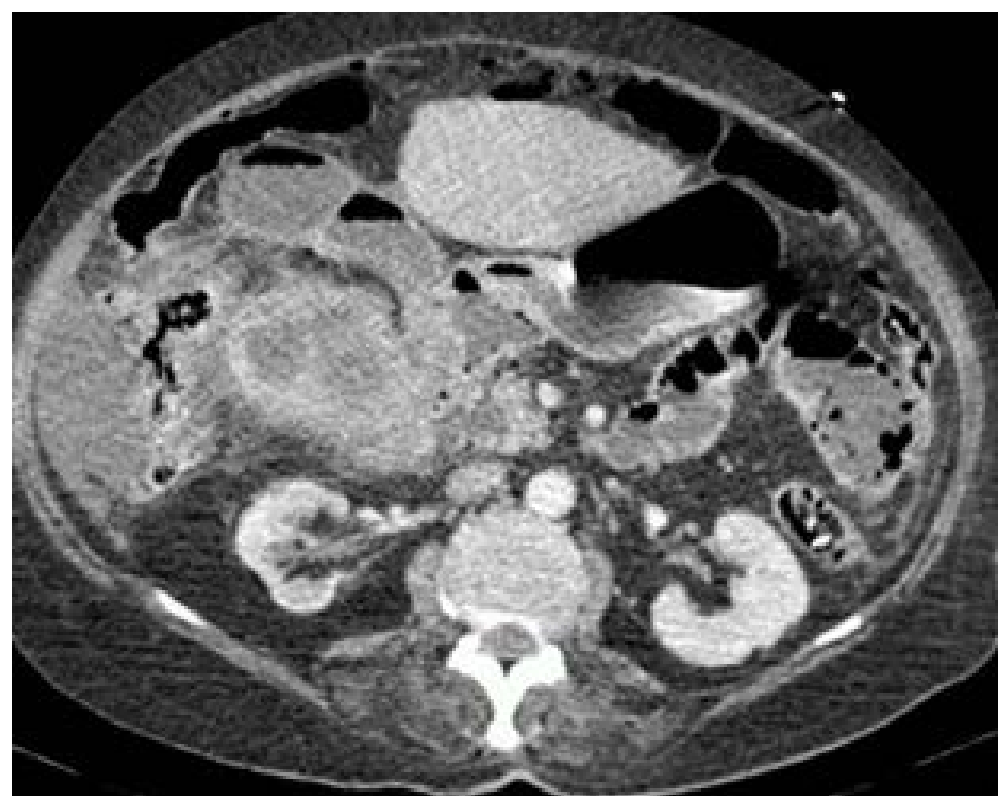

Figure 2. Free sub-diaphragmatic air was seen on the right, along with free intra-abdominal fluid in the peri-hepatic, sub-hepatic and lower abdominal spaces.

as dogs and foxes, which are also considered to be the definite hosts. Millions of eggs which are released from the adult form of the larva are passed into the feces [4] [5]. Intermediate hosts are herbivores that are infected by consuming contaminated feed. Humans are "accidental" intermediate hosts, usually infected through the consumption of contaminated vegetables/fruits. Once in human intestines, ingested eggs hatch releasing oncospheres which cross into the portal venous system before being lodged within hepatic sinusoids [2] [6]. It is this "mechanic" barrier that makes the liver the most common site (55\% - 70\%) for HC disease, which is followed in frequency of involvement by the lungs $(18 \%-35 \%)$. Simultaneous involvement of both organs maybe encountered in 5\% - 13\% of cases [2] [5]. Despite predilection to these organs, HC may potentially occur at any site of the body, including the brain, heart, kidneys, ureter, spleen, uterus, fallopian tubes, mesentery, pancreas, diaphragm and muscles [7]. In fact, 14\% - 19\% of intra-abdominal HCs are extra-hepatic.

Our patient's lesions were present in the liver and spleen. Patients with HC usually remain asymptomatic until a complication develops. Our patient who was asymptomatic was only diagnosed after presenting with a ruptured HC. Cyst rupture is the most important and frequent complication of HC. E. granulosus infections are particularly endemic in cattle and sheep breeders [2] [4] [6]. Our patient came from a village known for sheep herding, and she also had a history of raising sheep on a farm.

Three types of cyst rupture have been described in the literature: 1) contained rupture of an endocyst with an intact pericyst resulting in a membrane-like appearance of the collapsed endocyst on imaging; 2) communicating rupture with leakage of cyst contents into the biliary or bronchial tree, which is also the most frequently encountered type of rupture; 3) direct rupture usually involving the pericyst and endocyst with free spillage of cyst content into the pleural or peritoneal space [7] [8]. Peritoneal leakage may result in dissemination of the disease. Acute abdomen due to generalized peritonitis or may ensue.

Our patient's presentation to the emergency department was with an acute abdomen. The reported frequency of intraperitoneal rupture is $1 \%-8 \%$, with cyst rupture generally resulting in peritoneal irritation and shock [5] [6].

Important risk factors for HC rupture include age, cyst size of more than $10 \mathrm{~cm}$ and superficial localization. A cyst diameter of $20 \mathrm{~cm}$ at its widest point was probably the single most important cause of rupture in our patient, and peripheral localization of the cyst as well as a belated diagnosis after rupture were the main contributing factors to her death [9] [10].

Signs and symptoms of HC may vary depending on size and location, involved organ(s), and the presence of complications. Mechanic bowel obstruction as a result of rupture/fistulization of hepatic, splenic, pancreatic, 
mesenteric or retrovesical cysts into the gastrointestinal tract has been reported [2] [6].

Although cyst rupture is the most common complication of HC, rupture into the peritoneal space is rare but potentially serious. Intraperitoneal rupture of a HC may lead to a severe systemic anaphylactic reaction, with a reported rate of between $1 \%-12.5 \%$. A mortality rate due to anaphylaxis of up to $25 \%$ has been described in some studies. In fact, the patient's presenting complaint was abdominal pain, tachypnea, tachycardia. Our patient died despite surgical intervention following cyst rupture [11].

US and CT are important diagnostic tools for HC. The Gharbi classification which was introduced for US evaluation of HC provides details regarding cyst morphology [12]. Not only is US cheaper, but its widespread availability and high diagnostic yield makes it the imaging modality of choice. The cystic nature of a lesion, the presence of daughter vesicles and hydatiddebris can all be ascertained by US, making it ideal for reliably diagnosing HC [1] [12]. Although CT is considered complimentary to US in making a diagnosis of HC, it is the superior modality for the detection of peritoneal rupture. Not only does CT provide information regarding localization, size and number of cysts, but is also very useful in determining the relationship of a HC with the biliary tree or major blood vessels. CT is of particular use for the preoperative evaluation of multiple or complicated cysts which may help to optimize the surgical approach [2].

The mainstay treatment for HC remains surgery. In case of a ruptured HC, initial focus should be on the medical treatment of the ensuing allergic reaction, if present. This should be followed promptly by emergency surgery. The most important steps for successful surgical management of a ruptured cyst are sufficient washing of the peritoneal surface with a scolocidal agent and complete removal all cystic content. Giving patient albendazole at a dose of $10 \mathrm{mg} / \mathrm{kg} /$ day for 2 - 3 months after surgery has been shown to be safe and effective [1] [13]. Albendazole may also play an important prophylactic role in case of rupture. Our patient died despite effective peritoneal washing and complete removal of the cyst. Although percutaneous drainage has gained attention in recent years, surgery remains the most important treatment modality for HC [4] [5].

Ideally, a cyst should be dissected and removed without being ruptured, to prevent spread and contamination. Interventions for HC range from conservative approaches such as external drainage, partial cystectomy and cavity obliteration methods, to more radical surgical approaches such as pericystectomy and liver resection. The ideal approach remains a source of much debate. Reported operative mortality rate for perforated HC of the liver is $11.8 \%$, with post-surgical recurrence rates of $3 \%-10 \%$.

\section{Conclusion}

HC should be considered in the differential diagnosis in a patient presenting to the emergency department with abdominal pain. Radiologic modalities such as US and BT are important diagnostic tools, with US particularly offering details regarding cyst morphology. On its own, US is a reliable and efficient method for making a diagnosis. Giant HC may easily rupture, and intraperitoneal rupture is a potentially fatal complication. Such cases warrant initial follow-up in a resuscitation unit and admission for intensive care. Surgery should be not delayed in case of a ruptured HC due to the risk of developing anaphylactic shock.

\section{References}

[1] Majbar, A.M., Aalala, M., Elalaoui, M., Sabbah, F., Raiss, M., Hrora, A., et al. (2014) Asymptomatic İntra-Peritoneal Rupture of Hydatid Cyst of the Liver: Case Report. BMC Research Notes, 7, 114. http://dx.doi.org/10.1186/1756-0500-7-114

[2] Akalin, S., Kutlu, S.S., Caylak, S.D., Onal, O., Kaya, S. and Bozkurt, A.I. (2014) Seroprevalence of Human Cystic Echinococcosis and Risk Factors in Animal Breeders in Rural Communities in Denizli, Turkey. Journal of Infection in Developing Countries, 8, 1188-1194. http://dx.doi.org/10.3855/jidc.4343

[3] Salido, L., Mestre, J.L., Moya, J.L., Hernandez-Antolín, R. and Zamorano, J.L. (2015) Chronic Giant Hydatid Cyst Fistulized to the Left Ventricle: Long-Term Survival without Surgery. European Heart Journal Cardiovascular Imaging, 16, 798. http://dx.doi.org/10.1093/ehjci/jev042

[4] Galati, G., Sterpetti, A.V., Caputo, M., Adduci, M., Lucandri, G., Brozzetti, S., et al. (2006) Endoscopic Retrograde Cholangiography for İntrabiliary Rupture of Hydatid Cyst. American Journal of Surgery, 191, 206-210. http://dx.doi.org/10.1016/j.amjsurg.2005.09.014

[5] Arıbaş, B.K., Dingil, G., Köroğlu, M., Ungül, U. and Zaralı, A.C. (2011) Liver Hydatid Cyst with Transdiaphragmatic Rupture and Lung Hydatid Cyst Ruptured into Bronchi and Pleural Space. Cardiovascular and Interventional Radiology, 34, S260-S265. http://dx.doi.org/10.1007/s00270-009-9734-0 
[6] Dervisoglu, E., Topcu, S., Liman, S.T. and Yilmaz, A. (2008) Spontaneous Rupture of a Giant Diaphragmatic Hydatid Cyst into the İntrapleural Space. Medical Principles and Practice: International Journal of the Kuwait University, 17, 86-88. http://dx.doi.org/10.1159/000109598

[7] Ramia, J.M., De la Plaza, R., Quiñones, J., Veguillas, P. and Garcia-Parreño, J. (2011) Extremely High Values of CA 19-9 in Liver Hydatidosis and Frank Biliary Rupture. The Turkish Journal of Gastroenterology: The Official Journal of Turkish Society of Gastroenterology, 22, 548-550.

[8] Ok, U.Z., Ozkol, M., Kilimcioğlu, A.A., Dinç, G., Bayindir, P., Ostan, I., et al. P(2007) A Province-Based Study Using Sampling Method to İnvestigate the Prevalence of Cystic Echinococcosis among Primary School Children in Manisa, Turkey. Acta Tropica, 103, 116-122. http://dx.doi.org/10.1016/j.actatropica.2007.05.013

[9] Yang, Z.-Z., Li, Y., Liu, J., Li, K.-F., Yan, Y.-H. and Xiao, W.-D. (2013) Giant Biliary Cystadenoma Complicated with Polycystic Liver: A Case Report. World Journal of Gastroenterology, 19, 6310-6314.

[10] Moorthy, N., Ananthakrishna, R., Rajendran, R., Girish Gowda, S.L., Bhat, S.P.S. and Nanjappa, M.C. (2013) Giant Cardiac Hydatid Cyst: An Uncommon Cause of Cardiomegaly. Journal of the American College of Cardiology, 62, e145.

[11] Misra, A.P., Misra, R. and Kumar, A. (2013) Giant Cavernous Haemangioma of the Wandering Spleen. The Indian Journal of Surgery, 75, 54-55.

[12] Gharbi, H.A., Hassine, W., Brauner, M.W. and Dupuch, K. (1981) Ultrasound Examination of the Hydatic Liver. Radiology, 139, 459-463. http://dx.doi.org/10.1148/radiology.139.2.7220891

[13] Usluer, O., Ceylan, K.C., Kaya, S., Sevinc, S. and Gursoy, S. (2010) Surgical Management of Pulmonary Hydatid Cysts: İs Size an İmportant Prognostic İndicator? Texas Heart Institute Journal, 37, 429-434. 